\title{
THE CHARACTERIZATION PROBLEM FOR ENDOMORPHISM RINGS
}

\author{
J. L. GARCÍA \\ (Received 17 July 1989; revised 2 January 1990)
}

Communicated by B. J. Gardner

\begin{abstract}
We consider the problem of characterizing by abstract properties the rings which are isomorphic to the endomorphism ring $\operatorname{End}\left({ }_{R} F\right)$ of some free module $F$ over a ring $R$ in a given class $\mathscr{R}$ of rings. We solve this problem when $\mathscr{R}$ is any class of rings (by employing topological notions) and when $\mathscr{R}$ is the class of all the left Kasch rings (in terms of algebraic properties only).
\end{abstract}

1980 Mathematics subject classification (Amer. Math. Soc.) (1985 Revision): 16 A 65.

\section{Introduction}

As stated by Franzsen and Schultz in [3], the Characterization Problem is the following: given a class $\mathscr{R}$ of rings and a class $\mathscr{M}$ of modules over the rings in the class $\mathscr{R}$, describe in ring-theoretic terms the rings which are isomorphic to endomorphism rings $\operatorname{End}\left({ }_{R} M\right)$ for some $R$ in $\mathscr{R}$ and $M$ in $\mathscr{M}$. Significant advances were made (for some particular classes $\mathscr{R}$ and $\mathscr{M}$ ) by Wolfson [20] in the 50s; and by Metelli and Salce [14] and Liebert [11, $12,13]$ in the 70s. A more general result in this connection was obtained by Franzsen and Schultz [3, Theorem 3.2] in 1983: they provide a solution to the Characterization Problem for the class $\mathscr{K}$ of all the (locally) free $R$-modules over rings $R$ which satisfy the condition that each nonzero summand of a (locally) free $R$-module is indecomposable if and only if it is isomorphic to ${ }_{R} R$.

This research received partial support from the D.G.I.C.Y.T. (PB87-0703).

(C) 1991 Australian Mathematical Society 0263-6115/91\$A2.00+0.00 
The goal of this paper is to give an answer to the Characterization Problem under more general hypotheses. To this end, two different techniques are employed: first, the idea (which goes back to Liebert) of using both topological and algebraic conditions in the characterization of these rings; second, the consideration of categorical methods (and, in particular, category equivalences) as a tool to find necessary and sufficient conditions on a ring $E$ to be isomorphic to the endomorphism ring $\operatorname{End}\left({ }_{R} M\right)$ for a ring $R$ and generator ${ }_{R} M$. It is well-known that, if this happens, then the category $R$-mod is equivalent to a certain quotient category of $E$-mod. It turns out that this quotient category may be viewed as the full subcategory $E_{0}$-mod of all the unital and $E_{0}$-torsion-free left modules over a certain dense "subrng" $E_{0}$ of $E$ ("rng" means ring without a 1); namely, $E_{0}$ is the rng consisting of all the endomorphisms of $M$ that factor through some free module $R^{n}$. Thus we are naturally led to the question of finding also necessary conditions for having such an equivalence between $A$-mod and $R$-mod for a given $\mathrm{rng} A$ and ring $R$. The answer to this question makes it possible to find the way to solve the Characterization Problem for the class $\mathscr{K}$ of all the free modules over any class $\mathscr{R}$ of rings, thus generalizing the corresponding results of [13] and [3].

Specifically, we deal in Section 2 with the above stated problem of characterizing the rngs $A$ such that there exists a category equivalence between the already mentioned category $A$-mod and $R$-mod for a ring $R$. The answer to this question is related to some classical results about endomorphism rings of vector spaces, so we explain this relationship in some corollaries in Section 2. Now, for a given endomorphism ring $E=\operatorname{End}\left({ }_{R} M\right)$ let us denote by $\operatorname{fEnd}\left({ }_{R} M\right)=E_{0}$, the subrng of $E$ described in the previous paragraph. As a step toward the solution of the Characterization Problem, we consider in Section 3 the following special form of it: describe in ring-theoretic terms the rng fEnd $\left({ }_{R} M\right)$ for all free modules $M$ over any ring $R$. By means of the results of Section 2, we are able to give an answer to this problem and we subsequently study some particular cases in which $R$ is assumed to be, for example, left noetherian or a division ring.

The fourth section contains the main results. Theorem 4.2 gives the description of $\operatorname{End}\left({ }_{R} M\right)$ for ${ }_{R} M$ a free module and $R$ a ring in an arbitrary class $\mathscr{R}$ of rings: these are the rings $A$ that satisfy the following four conditions:

(i) $A$ contains a family of orthogonal idempotents $\left\{e_{i}\right\}_{i \in I}$ such that the left ideal of $A$ generated by these idempotents is also a right ideal;

(ii) for any pair $i, j \in I$ one has $A e_{i} \cong A e_{j}$;

(iii) $e_{i} A e_{i}$ is in the class $\mathscr{R}$ for any $i \in I$;

(iv) $A$ is Hausdorff and complete in the topology which has the family of 
all the right annihilators of the idempotents $e_{i}$ as a subbase of neighborhoods of 0 .

We again apply this to particular cases and obtain as consequences results in [13] or [3]. Finally, we also generalize [20, Theorem 7.5] in which the ring of the endomorphisms of a left vector space is characterized; Theorem 4.6 gives necessary and sufficient conditions for a ring $A$ to be isomorphic to End $\left({ }_{R} F\right)$ for a free module $F$ over a left Kasch ring $R$. These conditions are (i) $A$ contains a smallest dense ideal $A_{0}$; (ii) $A_{0}=\bigoplus_{I} A e_{i}$ for a set $I$ and a family of orthogonal idempotents $\left\{e_{i}\right\}$ such that all the $A e_{i}$ are isomorphic; (iii) if $J$ is a left ideal of $A$ which is maximal with respect to the property of not being dense, then $J$ has nonzero right annihilators; and (iv) $A$ is its own maximal left ring of quotients.

\section{Terminology and preliminary results}

Throughout the paper, rings will have an identity element 1 , while rngs are supposed not to have any, in general. Module will mean left module, unless stated otherwise. If $A$ is a $\mathrm{rng}$, a module ${ }_{A} M$ is unital when $A M=M$, that is, when $M$ has a spanning set; and ${ }_{A} M$ is finitely spanned, when it has a finite spanning set. We call ${ }_{A} M$ simple [4] when $M$ is unital, nonzero, and has no proper submodule other than 0 . We call ${ }_{A} M A$-torsion-free in case $A x=0$ implies $x=0$ for any $x \in M$; thus, $A$ is $A$-torsion-free if and only if $A$ is non-degenerate, in the terminology of [17]. $A$-MOD will denote the category of all the left $A$-modules, while $A$-mod will stand for the full subcategory of $A$-MOD containing all the unital and $A$-torsion-free left $A$-modules. If $A$ is a rng, $M$ is a left (respectively right) $A$-module and $X \subseteq M$, then $l_{A}(X)$ (respectively, $r_{A}(X)$ ) will denote the left (respectively right) annihilator of $X$ in $A$. The rng $A$ is said to be left $s$-unital [19] when $a \in A a$ for any $a \in A$. If $A$ is a rng and $e$ is an idempotent of $A$, then $\operatorname{End}\left({ }_{A} A e\right) \cong e A e$ in a natural way.

On the other hand, a module ${ }_{A} M$ is said to be intrinsically projective [2] when for every epimorphism $f: M^{n} \rightarrow L$ for $L$ a submodule of $M$, and every homomorphism $g: M \rightarrow L$, there exists $h: M \rightarrow M^{n}$ such that $f \circ h=g$.

It will be assumed that all categories appearing in this paper are additive categories and all functors (in particular, all equivalences) are additive functors. When dealing with endomorphism rings, the endomorphisms are supposed to act opposite scalars. For ring-theoretic terms not mentioned above, we refer the reader to [1]; for notions on torsion theories, which appear occasionally in the text, we refer to [18] (in particular, when $\mathscr{F}$ is a left 
Gabriel topology on a ring $R$, the associated quotient category $(R, \mathscr{F})$-mod will be supposed to be the full subcategory of $R$-mod consisting of all the $\mathscr{F}$ torsion-free and $\mathscr{F}$-injective modules); and, finally, [5] should be consulted for the topological terms.

In this paper, we shall be frequently dealing with the following situation: $R$ is a ring, $M$ is a left $R$-module, $E=\operatorname{End}\left({ }_{R} M\right)$ is the endomorphism ring of $M$ and $E_{0}=\operatorname{fEnd}\left({ }_{R} M\right)$ is the trace on $E$ of the derived context of ${ }_{R} M$ (see [15]), that is, $E_{0}$ consists of the endomorphisms of $M$ which factor through a free $R$-module of finite type. Now $E_{0}$ is a two-sided ideal of $E$ and, in some particular cases, (for example, if ${ }_{R} M$ is a generator) $E_{0}$ is idempotent by [21, Lemma 2.3]). We will usually consider $E_{0}$ as a rng in its own right. In particular, if ${ }_{R} M$ is a generator then $E_{0}$ is a non-degenerate and idempotent rng. Accordingly, $E_{0}$ is an object of the subcategory $E_{0^{-}}$ mod. If $R$ is a ring and $I$ is a set, $R F M_{I}(R)$ will stand for the ring of all the row-finite $I \times I$ matrices over $R$, while $F C_{I}(R)$ will denote the subrng of all those matrices that have finitely many nonzero columns (of course, if ${ }_{R} M=R^{(I)}$, then the former is isomorphic to $E$, while the latter is isomorphic to $E_{0}$ ). Also, the subrng of $F C_{I}(R)$ consisting of all the matrices which have a finite number of nonzero entries will be written $F M_{I}(R)$. All these notations will be employed without further reference.

We now state some useful facts which will be employed in the sequel. First, we extend the usual definition of matric units (see, for example, [17, Definition 1.1.2]).

Definimion 1.1. Let $A$ be a rng, $I$ a set. A family $\left\{e_{i j}\right\} \quad(i, j \in I)$ of elements of $A$ is called a family of matric units for the $\operatorname{rng} A$ when

(a) $e_{i j} e_{k h}=\delta_{j k} e_{i h}$, for all $i, j, k, h \in I$,

(b) for each $a \in A$, $a e_{i i}=0$ for almost all $i \in I$; and moreover, $a=\sum_{I} a e_{i i}$.

It is an easy matter to prove, similarly to [10, Proposition 5, page 52], the following result.

LEMMA 1.2. A rng $A$ has a family of matric units $\left\{e_{i j}\right\} \quad(i, j \in I)$ if and only if $A=\bigoplus_{I} A u_{i}$ for some set of orthogonal idempotents $\left\{u_{i}\right\}_{i \in I}$ of $A$ in such a way that all the $A u_{i}$ are isomorphic left ideals of $A$. Moreover, the $u_{i}$ may be taken to be $u_{i}=e_{i i}$.

Recall that a module ${ }_{R} M$ is called locally free if each finite set of elements of $M$ is contained in a (finitely generated) free direct summand of $M$. Plainly, a nonzero locally free module is a generator. On the other hand, a subrng $T$ of $E=\operatorname{End}\left({ }_{R} M\right)$ is said to be dense in $E$ when for each finite set $x_{1}, \ldots, x_{n}$ of elements of $M$ and every $s \in E$ there is some $t \in T$ such 
that $x_{i} t=x_{i}$ s for all $i=1, \ldots, n$. It is well-known that if $M$ is a nonzero locally free module, then $E_{0}$ is a dense subrng of $E$. In fact, the following density condition is easy to verify.

Proposition 1.3. Let ${ }_{R} M$ be a locally free module, $A$ a subrng of $E$. Then $A$ is dense in $E$ if and only if for every $s \in E_{0}$ we have that $s E_{0} \subseteq s A$.

Proof. This is straightforward.

COROLlary 1.4. Let $M$ be locally free, and let $A$ be a left ideal of $E$. Then $A$ is a dense subrng of $E$ if and only if $E_{0} \subseteq A$.

Proof. If $A$ is dense in $E$, then by Proposition 1.3 we have $E_{0}=E_{0}^{2} \subseteq$ $E_{0} A \subseteq A$, as $A$ is a left ideal of $E$.

\section{2. $q$-dense subrngs of endomorphism rings}

As we have just seen, if $A$ is a dense subrng of $E=\operatorname{End}\left({ }_{R} M\right),{ }_{R} M$ being a locally free module, then, by Proposition 1.3, we must have $E_{0} A=E_{0}$, since $E_{0}$ is a two-sided ideal of $E$. This suggests the following definition.

Definition 2.1. Let ${ }_{R} M$ be a generator, $E=\operatorname{End}\left({ }_{R} M\right), E_{0}=\operatorname{fEnd}\left({ }_{R} M\right)$. A subrng $A$ of $E$ will be called a $q$-dense subrng of $E$ whenever $E_{0} A=E_{0}$.

Moreover, we shall say that $A$ is a $q$-dense right ideal of $E_{0}$ in case $A$ is a right ideal of $E_{0}$ which is a $q$-dense subrng of $E$.

REMARK 2.2. As pointed out above, if $M$ is locally free and $A$ is a dense subrng of $E$, then $A$ is $q$-dense in $E$. Thus, since it is well-known that $F M_{I}(R)$ is a dense subrng of $R F M_{I}(R)$, and it is also a right ideal of $F C_{I}(R)$, then $F M_{I}(R)$ is a $q$-dense right ideal of $F C_{I}(R)$.

The converse is not true. For instance, let $R$ be an arbitrary ring, $M$ a free left $R$-module with an infinite countable basis, $M=R^{(\mathbb{N})}$; for every $n \in \mathbb{N}$, denote by $v_{n}$ the endomorphism of $M$ induced on each component $R_{m}=R$ of $M$ by the mapping $r \rightarrow r u_{n}$, where $\left\{u_{n}\right\}_{n \in \mathbb{N}}$ is the canonical basis of $M$. Let $A=\sum_{n \in \mathrm{N}} v_{n} E_{0}$ which is a right ideal of $E_{0}$. Moreover, if $e_{n}$ is the canonical projection of $M$ onto its $n$th component, we have $e_{n} v_{n}=e_{n}$, from which it follows that $E_{0}=E_{0} A$, that is, $A$ is a $q$-dense right ideal of $E_{0}$. But $A$ is not a dense subrng of $E$, as is easily seen.

In order to obtain a characterization of $q$-dense ideals in endomorphism rings, we need the following lemma. 
LEMMA 2.3. Let $S$ be a ring, $A$ a faithful and idempotent right ideal of $S$. Let $\mathscr{G}$ be the left Gabriel topology of $S$ given by $\mathscr{G}=\left\{I \subseteq{ }_{S} S \mid A \subseteq I\right\}=$ $\left\{I \subseteq \subseteq_{S} S \mid S A \subseteq I\right\}$, and let $(S, \mathscr{G})$-mod be the corresponding quotient category considered as a full subcategory of $S$-mod. Let $L$ be the functor $L: S$-mod $\rightarrow A$-MOD given on objects by $L\left({ }_{S} X\right)=A X$. Then $L$ induces an equivalence between the subcategories $(S, \mathscr{G})$-mod and $A$-mod.

Proof. It is easy to verify that if ${ }_{S} X$ belongs to $(S, \mathscr{G})$-mod, then $A X$ belongs to $A$-mod, since ${ }_{S} X$ is $\mathscr{G}$-torsion-free. Thus $L$ restricts to an additive functor $L^{\prime}:(S, \mathscr{G})$-mod $\rightarrow A$-mod, which is a faithful functor, because if $\alpha: X \rightarrow Y$ is a morphism in $(S, \mathscr{G})$-mod and $\alpha(A X)=0$, then $A \alpha(X)=S A \alpha(X)=0$ and $\alpha(X)$ is $\mathscr{G}$-torsion, so $\alpha(X)=0$ and $\alpha=0$. Now, let $\beta: A X \rightarrow A Y$ be an $A$-homomorphism with $X$ and $Y$ in $(S, \mathscr{G})$ mod. For each $x \in X$, define $\beta_{x}: A \rightarrow A Y$ by $\beta_{x}(a)=\beta(a x)$. Then $\beta_{x}$ is an $A$-homomorphism which can be extended to $\alpha_{x}: S A \rightarrow Y$ in the following way: if $s \in S, a \in A$, put $\alpha_{x}(s a)=s \beta_{x}(a)$. Then $\alpha_{x}$ is well-defined because $Y$ is $\mathscr{G}$-torsion-free; and it is in fact an $S$-homomorphism. Then $\alpha_{x}$ may be extended to an $S$-homomorphism from $S$ to $Y$ because $Y$ is $\mathscr{G}$-injective and $S / S A$ is $\mathscr{G}$-torsion, and hence it follows that there is some $y=y(x)$ such that $\beta_{x}(a)=a y$ for each $a \in A$. This shows that there exists $g: X \rightarrow Y$ such that $g(a x)=a g(x)=\beta(a x)$, for all $a \in A$, and all $x \in X$. But $g$ is also an $S$-homomorphism because $A$ is a right ideal of $S$ and $Y$ is $\mathscr{G}$-torsion-free. Since $g$ is an extension of $\beta$, this proves that $L^{\prime}$ is a full functor.

To complete the proof it only remains to show that for any $A$-module $X$ in $A$-mod, $X=A N$ for some ${ }_{s} N$ in $(S, \mathscr{G})$-mod. In fact, it suffices to prove that this happens for some ${ }_{S} N$ which is $\mathscr{G}$-torsion-free, because if ${ }_{S} N$ is $\mathscr{G}$-torsion-free and $N^{\prime}$ is the localization of $N$, then $(S A) N=(S A) N^{\prime}$ and $A(S A) N=A N=A N^{\prime}$. Now, given ${ }_{A} X$ unital and $A$-torsion-free, take $X^{\prime}=\operatorname{Hom}_{A}(A, X)$. By identifying each element $x$ of $X$ with right multiplication by $x$, we may assume that $X$ is a submodule of $X^{\prime}$ and, since $X$ is unital, $A X^{\prime}=X$. On the other hand, each element $s \in S$ can be interpreted as an $A$-endomorphism of $A$, as $A$ is a faithful right ideal of $S$. In this way, $X^{\prime}$ is a left $S$-module. Finally, $X^{\prime}$ is $\mathscr{G}$-torsion-free because $X$ is $A$-torsion-free and thus we are done.

The reason for our interest in Definition 2.1 lies in the next result (the equivalence between (i) and (ii) appears already in [6, Theorems 4 and 5], but we thought it better to give another proof below for the convenience of the reader).

TheOREM 2.4. Let $A$ be a non-degenerate and idempotent $r$ ing and $R a$ ring. The following conditions are equivalent. 
(i) There exists a generator ${ }_{R} M$ of $R$-mod such that $A$ is isomorphic to a q-dense right ideal $T$ of the rng $E_{0}:=\operatorname{fEnd}\left({ }_{R} M\right)$.

(ii) There is an equivalence of categories $F: R$-mod $\rightarrow A$-mod.

(iii) There is a module ${ }_{A} N$ such that $N$ is finitely spanned, intrinsically projective and generates $A$, and $R \cong \operatorname{End}\left({ }_{A} N\right)$.

Proof. (i) $\Rightarrow$ (ii). Assume that ${ }_{R} M$ is a generator of $R$-mod with $E=$ $\operatorname{End}\left({ }_{R} M\right), E_{0}=\operatorname{fEnd}\left({ }_{R} M\right)$ and $T$ an idempotent right ideal of $E_{0}$ such that $E_{0} T=E_{0}, T \cong A$, so that $T E_{0}=T$. By the Gabriel-Popescu Theorem [18, Theorem X.4.1], the functor $\operatorname{Hom}_{R}(M,-): R$-mod $\rightarrow E$-mod is full and faithful and induces an equivalence of categories between $R$-mod and the smallest quotient category of $E$-mod which contains all modules of the form $\operatorname{Hom}_{R}(M, X)$. By [9, Theorem 1.7 and Proposition 2.5], this category is the quotient category of $E$-mod with respect to the Gabriel topology $\mathscr{G}$ of the left ideals of $E$ that contain $E_{0}$ or, equivalently, $T$. Since ${ }_{E} T$ is a faithful right ideal of $E$ (because $E_{0}$ is clearly faithful), we may apply Lemma 2.3 to $T$ and $(E, \mathscr{G})$-mod, so that we get that $(E, \mathscr{G})$-mod and $T$-mod are equivalent categories. The isomorphism $T \cong A$ gives then (ii).

(ii) $\Rightarrow($ i). Let $F: R$-mod $\rightarrow A$-mod be an equivalence and let us put $S=\operatorname{End}\left({ }_{A} A\right)$. Then $A_{S}$ is a right ideal of $S$ (because each $a \in A$ may be considered as right multiplication by $a$, and $A$ is non-degenerate) and in fact $A_{S}$ is faithful because $A=A^{2}$. Let $\mathscr{H}$ be the left Gabriel topology of $S$ given by $\mathscr{H}=\left\{I \subseteq{ }_{S} S \mid S A \subseteq I\right\}=\left\{I \subseteq{ }_{S} S \mid A \subseteq I\right\}$. Then $(S, \mathscr{H})$-mod and $A$-mod are equivalent categories by Lemma 2.3. By composing $F$ with this equivalence we obtain another equivalence $U: R-\bmod \rightarrow(S, \mathscr{H})$-mod. A direct computation shows that $S=\operatorname{End}\left({ }_{A} A\right) \cong \operatorname{End}\left({ }_{S A} S A\right)=\operatorname{End}\left({ }_{S} S A\right)$, so we deduce from [18, page 198] that $S$ belongs to the quotient category $(S, \mathscr{H})$-mod. By applying now [9, Theorem 1.19] to the equivalence $U$ we see that there is a left $R$-module $M$ such that ${ }_{R} M$ is a generator, $S \cong E=$ $\operatorname{End}\left({ }_{R} M\right)$, the functor $U$ is given, up to equivalence, by $\operatorname{Hom}_{R}(M,-)$, and, modulo the above isomorphism $S \cong E$, the topology $\mathscr{H}$ is the left topology $\mathscr{G}$ on $E$ given by $\mathscr{G}=\left\{I \subseteq{ }_{E} E \mid E_{0} \subseteq I\right\}$. This shows that the isomorphism $\phi: S \rightarrow E$ restricts to an isomorphism between $S A$ and $E_{0}$ and hence $\phi(A)=T$ is an idempotent right ideal of $E$ contained in $E_{0}$, satisfying $E_{0} T=E_{0}, T E_{0}=T$.

(ii) $\Rightarrow$ (iii). This is immediate, if we take $N=F(R)$, since then $N$ is a finitely generated projective generator in $A$-mod and $R \cong \operatorname{End}\left({ }_{A} N\right)$.

(iii) $\Rightarrow$ (ii). By (iii), ${ }_{A} N$ is a finitely generated generator of the category $A$-mod. Again by the Gabriel-Popescu Theorem we deduce that $A$-mod is equivalent, by means of the functor $\operatorname{Hom}_{A}(N,-)$, to a certain quotient category of $R$-mod, $(R, \mathscr{F})$-mod, in such a way that all the $R$-modules of the 
form $\operatorname{Hom}_{A}(N, X)$ (and, in particular, ${ }_{R} R$ ) belong to $(R, \mathscr{F})$-mod. Let now $I$ be a finitely generated left ideal of $R, g: N^{(I)} \rightarrow N$ the induced homomorphism and put $L:=\operatorname{Im} g$. From the facts that $N$ is intrinsically projective and $I$ is finitely generated we deduce by [2, Lemma 2] that $I=\operatorname{Hom}_{A}(N, L)$, and hence that $I$ is also in $(R, F)$-mod. But $R$ is a finitely generated object of $(R, \mathscr{F})$-mod because ${ }_{A} N$ is finitely generated, and thus $\mathscr{F}$ must have a basis of finitely generated left ideals [18, Proposition XIII.1.1]. This means that $\mathscr{F}$ is trivial, for $I \in \mathscr{F}$ and $I \in(R, \mathscr{F})$-mod imply $I=R$. Therefore $(R, \mathscr{F})$-mod coincides with $R$-mod and this proves (ii).

Some facts which do not actually appear in the statement of Theorem 2.4 are nevertheless obtained in the course of the proof above. We include now with a couple of useful results which will be used later.

COROLlaRY 2.5. Let $A$ be a non-degenerate and idempotent rng and $R a$ ring.

(a) If condition (i) in Theorem 2.4 holds, then the equivalence $F$ of (ii) can be chosen so that $F\left({ }_{R} M\right) \cong A$. Therefore $E=\operatorname{End}\left({ }_{R} M\right) \cong S=\operatorname{End}\left({ }_{A} A\right)$. Moreover, this isomorphism extends the isomorphism in (i) between $T$ and A.

(b) If (ii) of Theorem 2.4 holds for a given equivalence $F$, then one can choose ${ }_{R} M$ satisfying (i) and such that there is an isomorphism between $E=$ $\operatorname{End}\left({ }_{R} M\right)$ and $S=\operatorname{End}\left({ }_{A} A\right)$ which is an extension of the isomorphism in (i) between $T$ and $A$.

REMARK 2.6. The equivalence of (ii) and (iii) of Theorem 2.4 is very similar to part of [4, Theorem 1.1], the only difference being that in [4] a complete additive subcategory of $A$-MOD substitutes $A$-mod (but $A$-mod need not be such, even if it satisfies the conditions in Theorem 2.4), while here $A$ is assumed to be idempotent and non-degenerate. On the other hand, the conditions on the module ${ }_{A} N$ are also slightly (and accordingly) different in both cases.

Motivated by Theorem 2.4, our first approach to the Characterization Problem will be as follows: for classes $\mathscr{R}$ and $\mathscr{M}$ as already stated, find necessary and sufficient conditions for an abstract rng $A$ to be isomorphic to a $q$-dense right ideal of some $\operatorname{fEnd}\left({ }_{R} M\right)$, with $R \in \mathscr{R}$ and $M \in \mathscr{M}$. More specifically, we consider the following case: $\mathscr{R}$ is the class of division rings, $\mathscr{M}$ that of all the nonzero modules over rings in $\mathscr{R}$ ( $\mathscr{R}$ could equally well be taken to be the class of simple artinian rings). In order to solve this problem, let us recall the following definition (which is due to Dieudonné). 
Definition 2.7. A $\mathrm{rng} A$ is quasi-simple when ${ }_{A} A$ is a direct sum of isomorphic minimal left ideals.

Proposition 2.8. Let $A$ be a rng, $A^{2} \neq 0$. The following conditions are equivalent.

(i) $A$ is quasi-simple.

(ii) There is a division ring $D$ and a left $D$-vector space $V$ such that $A$ is isomorphic to a q-dense right ideal of $\operatorname{fEnd}\left({ }_{D} V\right)$.

(iii) There is a division ring $D$ and a left $D$-vector space $V$ such that $A$ is isomorphic to a nonzero right ideal of $\operatorname{fEnd}\left({ }_{D} V\right)$.

In particular, quasi-simple rngs $A$ such that $A^{2} \neq 0$ are idempotent.

Proof. We begin by establishing the final sentence of the proposition. Let $A$ be quasi-simple and $A^{2} \neq 0$. Assume that $A=\bigoplus_{I} S_{i}$ with all the $S_{i}$ isomorphic minimal left ideals of $A$. Then $A S_{j} \neq 0$ for some $j \in I$ so $A S_{j}=S_{j}$ and $S_{j}$ is simple. Hence, so are all the $S_{i}$ and thus $A^{2}=A$. The same argument shows that $r_{A}(A)=0$, so that $A$ is non-degenerate.

(i) $\Rightarrow$ (ii). We have just seen that $A$ is idempotent and non-degenerate and, on the other hand, each simple left ideal of $A$ is a finitely spanned (projective) generator of $A$-mod. By Schur's lemma and Theorem 2.4 there is a division ring $D$ and a nonzero left $D$-vector space $V$ such that $A$ is isomorphic to a $q$-dense right ideal of $E_{0}=\operatorname{fEnd}\left({ }_{D} V\right)$.

(ii) $\Rightarrow$ (i). We may assume that $A$ is a $q$-dense right ideal of $E_{0}=$ fEnd $\left({ }_{D} V\right)$ for some nonzero $D$-vector space $V$. It is clear that $E_{0}$ is right $s$-unital, from which it follows that $A E_{0}=A$. This implies that $A$ is idempotent (since $A=A E_{0}=A\left(E_{0} A\right)=A^{2}$ ) and non-degenerate (because $E_{0}$ is non-degenerate as well). Therefore we may apply Theorem 2.4 to obtain that $A$-mod is equivalent to $D$-mod and so $A$-mod has a simple generator. Consequently, $A$ is a direct sum of isomorphic simple $A$-modules, that is, $A$ is quasi-simple.

(ii) $\Rightarrow$ (iii) is obvious.

(iii) $\Rightarrow$ (ii). If $A$ is a nonzero right ideal of $E_{0}=\operatorname{fEnd}\left({ }_{D} V\right)$, then $E_{0} A$ is a nonzero two-sided ideal of $E_{0}$ and, since $E_{0}$ is a simple $\mathrm{rng}, E_{0} A=E_{0}$. Thus $A$ is a $q$-dense right ideal of $E_{0}$.

It is well-known that a quasi-simple rng need not be simple. Regarding this, we can derive from Proposition 2.8, the following result.

Proposition 2.9. Let $A$ be a quasi-simple rng. Then $A$ is simple if and only if $A^{2} \neq 0$ and $l_{A}(A)=0$. 
Proof. If $A$ is simple, then $A^{2} \neq 0$ by definition and $l_{A}(A)=0$, as it is a proper ideal of $A$. To show the converse we may assume, by Proposition 2.8, that $A$ is a nonzero idempotent right ideal of $E_{0}=\operatorname{fEnd}\left({ }_{D} V\right)$, for $D$ a division ring. If $A$ has a nonzero two-sided ideal $I$, then $I A \neq 0$ by the assumption $l_{A}(A)=0$. Then $I A$ is a nonzero right ideal of $E_{0}$. Now, $E_{0}(I A) \neq 0$ (as $E_{0}$ is non-degenerate) and so $E_{0} I A$ is a nonzero two-sided ideal of the simple $\mathrm{mg} E_{0}$, and hence $E_{0} I A=E_{0}$. Then $I \supseteq A I A=$ $A E_{0} I A=A E_{0}=A$, which shows that $I=A$ and $A$ is a simple rng.

It is clear that quasi-simple simple rngs are precisely the simple rngs with minimal left ideals. In the older literature, these rngs are known to be exactly the (left) primitive rngs which coincide with their (left) socle. But, in fact, the condition $l_{A}(A)=0$ for a nonzero quasi-simple $\mathrm{rng} A$ means exactly that each simple left ideal of $A$ is faithful, that is, $A$ is left primitive. On the other hand, these same rngs are characterized in [10, page 74] as those subrngs $A$ of $E_{0}=\operatorname{fEnd}\left({ }_{D} V\right.$ ) (for some division ring $D$ and vector space $V$ ) such that there exists a submodule $L^{\prime}$ of the dual space $V^{*}$ satisfying that $L^{\prime}$ annihilates only the 0 element of $V$, and $A$ consists of all the endomorphisms of $V$ of the form $\sum_{i=1}^{n} \varphi_{i} \lambda_{i}$, where $\varphi_{i} \in L^{\prime}$ and $\lambda_{i}: D \rightarrow$ $V$. Now, it is easy to see that every nonzero right ideal $A$ of $E_{0}$ is obtained from some submodule $L^{\prime}$ of $V^{*}$ in the same way described above; then the condition $l_{A}(A)=0$ is equivalent under this frame to the fact that the only element of $V$ annihilated by $L^{\prime}$ is zero.

\section{The rng of the finite endomorphisms}

We consider now the problem, for a given $\operatorname{rng} A$, of when $A$ is just isomorphic to $f \operatorname{End}\left({ }_{R} M\right)$, that is, the finite endomorphism rng of some generator $M$ for a ring $R$. We have the following result.

Theorem 3.1. Let $A$ be a rng. The following conditions are equivalent.

(a) There is a ring $R$ and a generator ${ }_{R} M$ such that $A$ is isomorphic to $E_{0}=\mathrm{fEnd}\left({ }_{R} M\right)$.

(b)

(i) There exists a finitely spanned and intrinsically projective left $A$-module $N$ such that $N$ generates $A$; and

(ii) $A$ is a left ideal in its endomorphism ring.

Moreover, $N$ and $R$ can be chosen in either case so that $R \cong \operatorname{End}\left({ }_{A} N\right)$.

Proof. (a) $\Rightarrow$ (b). Since, by (a), ${ }_{R} M$ is a generator, we see that $E_{0}$ is a non-degenerate and idempotent rng and hence, by Theorem 2.4 , the hypoth- 
esis implies already condition (i) with $R \cong \operatorname{End}\left({ }_{A} N\right)$. On the other hand, let $S=\operatorname{End}\left({ }_{A} A\right), E=\operatorname{End}\left({ }_{R} M\right)$. By Corollary 2.5 , there is an isomorphism $\phi: E \rightarrow S$ such that $\phi\left(E_{0}\right)=A$. Since $E_{0}$ is an ideal of $E, A_{S}$ is also a two-sided ideal of $S$, which proves (ii).

(b) $\Rightarrow$ (a). Note first that condition (ii) implies that $A$ is non-degenerate, for if $A x=0$ for $x \in A$, then the endomorphism of $A$ consisting of right multiplication by $x$ is zero, whence $x=0$. Then, let $N$ be as stated in (i); there exists an epimorphism $p: N^{(I)} \rightarrow A$ and also $A N=N$, from which it follows that $A N^{(I)}=(A N)^{(I)}=N^{(I)}$ and thus $A=p\left(N^{(I)}\right)=p\left(A N^{(I)}\right)=A^{2}$, so that $A$ is also idempotent.

Therefore we can apply Theorem 2.4 with $R=\operatorname{End}\left({ }_{A} N\right)$ to get that $A$ is isomorphic to a $q$-dense right ideal $T$ of $E_{0}=\operatorname{fEnd}\left({ }_{R} M\right), M$ being a generator for $R$-mod. Now, if we let $S=\operatorname{End}\left({ }_{A} A\right)$ we see, by Corollary 2.5 , that there is an isomorphism $\phi: S \rightarrow E$ with $\phi(A)=T$. By (ii) $A$ is a left ideal of $S$ and hence $T$ is also a left ideal of $E$, from which it follows that $E_{0} T=T$ and we are done.

As an application of the foregoing theorem we can consider the case of $R$ being a division ring.

Corollary 3.2. Let $A$ be a rng. Then $A$ is isomorphic to $\operatorname{fEnd}\left({ }_{D} V\right)$ for some nonzero vector space $V$ over a division ring $D$ if and only if the following two conditions are satisfied:

(i) $A$ is a nonzero quasi-simple rng,

(ii) $A$ is a left ideal in its endomorphism ring.

Proof. This a direct consequence of Theorems 2.4 and 3.1, along with Proposition 2.8.

REMARK 3.3. In [20, Theorem 6.2], the rngs of Corollary 3.2 are characterized as the simple rngs $A$ with minimal right ideals such that every proper left ideal of $A$ has a nonzero right annihilator. We want to give a short proof of this equivalence by means of our previous results. We have already seen that the conditions in Corollary 3.2 as well as those in [20, Theorem 6.2] imply in each case that $A$ is a ( $q$-dense) right ideal of $E_{0}:=\operatorname{fEnd}\left({ }_{D} V\right)$, and, through the equivalence $D$-mod $\cong A$-mod, $\operatorname{End}\left({ }_{D} V\right)=E \cong S=\operatorname{End}\left({ }_{A} A\right)$. Thus, all we have to show is that, under these hypotheses, (ii) of Corollary 3.2 is equivalent to the condition that $r_{A}(I)=0$ implies $I=A$, for any left ideal $I$ of $A$. First, if $A=E_{0}$ and $r_{A}(I)=0$, then $V I=V$ and by [8, page 93], $E_{0}=I$. Conversely, if $A \neq E_{0}$ let $\alpha E_{0}$ be a simple right ideal of $E_{0}$ satisfying $\alpha E_{0} \cap A=0$, and let $J=l_{E_{0}}(\alpha)$, a left ideal of $E_{0}$; it is plain that $r_{E_{0}}(J)=\alpha E_{0}$ and hence $r_{E_{0}}(J) \cap A=r_{A}(J)=0$, so $r_{A}(A J)=0$ 
(because $\left.r_{E_{0}}(A) \subseteq r_{E_{0}}\left(E_{0} A\right)=0\right)$ and $A J=A$ by the hypothesis. Therefore $A \alpha=0$ and $\alpha=0$, a contradiction.

In order to study the case when ${ }_{R} M$ is a free module, we give the following characterization.

Proposition 3.4. Let $A$ be a non-degenerate rng. Then $A$ has a family $\left\{e_{i j}\right\} \quad(i, j \in I)$ of matric units if and only if there is a ring $R$ such that $A$ is isomorphic to a q-dense right ideal $T$ of the finite column matrix ring of order $I$ of $R, F C_{I}(R)$, and $T$ contains all the matrices with a finite number of nonzero entries.

Moreover, $R$ can then be chosen so that $R \cong \operatorname{End}\left({ }_{A} A e_{i i}\right) \cong e_{i i} A e_{i i}$ for any $i \in I$.

Proof. Let $A$ be non-degenerate with a family $\left\{e_{i j}\right\},(i, j \in I)$ of matric units. Put $e_{i}:=e_{i i}$ for any $i \in I$ and $N=A e_{j}$ for some $j$. By Lemma 1.2, $A=\bigoplus_{I} A e_{i}$ and all the $A e_{i}$ are isomorphic, so $N$ is plainly a generator of $A$ mod. If one sets $R=\operatorname{End}\left({ }_{A} N\right)$, then, by the Gabriel-Popescu Theorem, there exists a category equivalence $\operatorname{Hom}_{A}(N,-): A$-mod $\rightarrow(R, F)$-mod, for a certain left Gabriel topology $F$ of $R$. In this equivalence $N$ corresponds to $R$ and $A$ corresponds to $\operatorname{Hom}_{A}(N, A) \cong R^{(I)}=F$, a free module. Therefore there is an induced isomorphism $\phi: S \rightarrow E$, where $S=\operatorname{End}\left({ }_{A} A\right)$, $E=\operatorname{End}\left({ }_{R} F\right)$. Consider $A$, as usual, as a right ideal of $S$; then $\phi(A)=B$ is an idempotent right ideal of $E$. Since the elements of $A$ correspond to endomorphisms of $A$ which factor through some $N^{k}$, we have that the elements of $B$ are endomorphisms of ${ }_{R} F$ which factor through some $R^{k}$, that is, $B \subseteq E_{0}=\operatorname{fEnd}\left({ }_{R} F\right)$. Moreover, each $e_{i}: A \rightarrow A$ is taken by the equivalence to the projection $\pi_{i}$ of $F=R^{(I)}$ onto its $i$ th component. Then $E_{0}=\bigoplus_{I} E_{0} \pi_{i}$ verifies $E_{0} B=E_{0}$ so $B$ is a $q$-dense right ideal of $E_{0}$. Finally, let $B_{0}$ be the subrng of $E$ consisting of all the endomorphisms $\sigma$ of $F$ such that $\pi_{i} \sigma=0$ for almost all $i \in I$. Then $B_{0}=\bigoplus_{I} \pi_{i} E_{0} \subseteq B$. Now, if we identify $E$ with $R F M_{I}(R)$, then $E_{0}$ corresponds to $F C_{I}(R)$, and $B_{0}$ corresponds to $F M_{I}(R)$, the subrng of all the matrices of order $I$ with a finite number of nonzero entries. Then, the subrng $T$ corresponding to $B$ satisfies the statement of the proposition.

Conversely, let $A \cong B, B_{0} \subseteq B \subseteq E_{0}$ with $B$ a $q$-dense right ideal of $E_{0}$, where we use the same notation as in the first part of the proof. Thus $\pi_{j} \in B_{0}$ for any $j \in I$. Since $B$ is a right ideal of $E_{0}$ we obtain that $B \supseteq \bigoplus_{I} B \pi_{i}$; on the other hand, for any $\alpha \in E_{0}$ we have that $\alpha=\sum_{C} \alpha \pi_{i}$ for some finite subset $C$ of $I$, and hence $B \subseteq \bigoplus_{I} B \pi_{i}$. Also, for any $i, j \in I$ we have an $E_{0}$-isomorphism between $E_{0} \pi_{i}$ and $E_{0} \pi_{j}$, which restricts to a $B$ isomorphism $B \pi_{i} \cong B \pi_{j}$. By Lemma $1.2, B$ has a family of matric units and the isomorphism $A \cong B$ completes the proof. 
Corollary 3.5. Let $A$ be a rng. The following conditions are equivalent.

(a) There exist a ring $R$ and a free left $R$-module $F$ such that $A$ is isomorphic to $\operatorname{fEnd}\left({ }_{R} F\right)$.

(b) $A$ has a family of matric units and $A$ is a left ideal in its endomorphism ring.

(c) $A$ is a left ideal of its endomorphism ring and $A=\bigoplus_{I} A e_{i}$, where $\left\{e_{i}\right\}_{i \in I}$ is a family of orthogonal idempotents of $A$ satisfying $A e_{i} \cong$ $A e_{j}$ for any $i, j \in I$.

Moreover, $R$ and the $e_{i}$ can be chosen so that $R \cong e_{i} A e_{i}$ for any $i \in I$.

Proof. The equivalence (b) $\Leftrightarrow$ (c) is clear from Lemma 1.2. That $(a) \Rightarrow(b)$ is immediate from Proposition 3.4 and Theorem 3.1. Finally, (b) implies that $A$ is non-degenerate and hence it is isomorphic to a $q$-dense right ideal $T$ of $E_{0}=\operatorname{fEnd}\left({ }_{R} F\right)$ for some ring $R$ and free module ${ }_{R} F$, also from Proposition 3.4. Since (b) implies also that $A$ is idempotent, we may apply Theorem 2.4 (and Corollary 2.5) to $T$ and obtain an isomorphism $\phi: \operatorname{End}\left({ }_{A} A\right)=S \rightarrow$ $E=\operatorname{End}\left({ }_{R} F\right)$ with $\phi(A)=T$. This shows that $T$ is a left ideal of $E$, and hence $T=E_{0}$ because it is $q$-dense in $E_{0}$.

We now want to characterize rngs which are isomorphic to $\operatorname{fEnd}\left({ }_{R} F\right)$ for a free module $F$ over a ring $R$ such that ${ }_{R} R$ is a direct sum of indecomposable left ideals. Recall that an idempotent $e$ of a ring $R$ is said to be finite in case it is the sum of finitely many orthogonal primitive idempotents. Then we have

THEOREM 3.6. Let $A$ be a rng. The following conditions are equivalent.

(a) $A$ is isomorphic to $\operatorname{fEnd}\left({ }_{R} F\right)$ for a free left $R$-module $F$ and a ring $R$ such that ${ }_{R} R$ is a direct sum of indecomposable left ideals.

(b)

(i) $A$ has a family of matric units $\left\{e_{i j}\right\}_{i, j \in I}$ such that each $e_{i}:=e_{i i}$ is a finite idempotent; and

(ii) $A$ is a left ideal in its endomorphism ring.

Proof. We already know from Corollary 3.5 that (a) implies (i) and (ii) except for the condition that the $e_{i}$ are finite. But if $N=A e_{i}$ for some fixed $i \in I$, then $R \cong \operatorname{End}\left({ }_{A} N\right)$ and the category equivalence $R$-mod $\cong$ $A$-mod gives us that, since ${ }_{R} R$ has an indecomposable decomposition, so has $N$ and hence $A e_{i}=\bigoplus_{k=1}^{r} L_{k}$ in such a way that $e_{i}=u_{1}+\cdots+u_{r}$ and the $u_{k}$ are orthogonal primitive idempotents. Conversely, Corollary 3.5 shows that (b) implies that $A$ is isomorphic to $\operatorname{fEnd}\left({ }_{R} F\right)$, where $F$ is free and $R \cong \operatorname{End}\left({ }_{A} A e_{i}\right)$; moreover, $A e_{i}$ corresponds to $R$ in the equivalence $R$-mod $\cong A$-mod, so that the assumption that $A e_{i}$ is a finite direct sum of 
indecomposable $A$-modules proves that ${ }_{R} R$ satisfies the same, and hence (a) holds.

We now consider a special case of the preceding theorem. Let us define, for any rng $A$ a transitive relation by setting $a \leq b$ if and only if there is $t \in A$ such that $t b=a$. We write $a<b$ whenever $a \leq b$ but not $b \leq a$. An element $s \in A$ will be called noetherian when there is no infinite chain $a_{1}<a_{2}<\cdots<a_{n}<\cdots$ of elements of $A$ with $a_{n} \leq s$ for each $n$. We then have the following result.

Corollary 3.7. Let $A$ be a rng. The following conditions are equivalent.

(a) There exists a left noetherian ring $R$ and a non-finitely generated free module ${ }_{R} F$ such that $A \cong \operatorname{fEnd}\left({ }_{R} F\right)$.

(b)

(i) $A$ has an infinite family of matric units $\left\{e_{i j}\right\}$ such that each $e_{i}:=e_{i i}$ is a noetherian idempotent; and

(ii) $A$ is a left ideal in its endomorphism ring.

(c)

(i) $A$ has an infinite family of matric units;

(ii) $A$ is a left ideal in its endomorphism ring, and

(iii) $A$ is left s-unital.

Proof. (a) $\Leftrightarrow$ (b). By Theorem 3.6, all that is left to do is to see that, under the hypothesis that the equivalent conditions of Theorem 3.6 hold, $R$ is left noetherian if and only if $e_{i}$ is a noetherian idempotent. Note that the relation $a<b$ for $a, b \in A \subseteq \operatorname{End}\left({ }_{R} F\right)$ means exactly that $\operatorname{Im} a \subset \operatorname{Im} b$ (where $\subset$ is strict inclusion). Since $A e_{i}$ can be viewed as $\operatorname{Hom}_{R}(F, R)$, we see that if $R$ is left noetherian, then $e_{i}$ must be a noetherian idempotent. Conversely, assume that $R$ is not left noetherian and let

$$
L_{1} \subset L_{2} \subset \cdots \subset L_{n} \subset \cdots
$$

be an infinite proper ascending chain of finitely generated left ideals of $R$. We can obtain from this chain $R$-homomorphisms $\alpha_{1}, \alpha_{2}, \ldots, \alpha_{n}, \ldots$ of $A e_{i}=\operatorname{Hom}_{R}(F, R)$ in such a way that, for each $n, \alpha_{n} \leq e_{i}$ and $\alpha_{n}<$ $\alpha_{n+1}$ (simply by taking $\operatorname{Im} \alpha_{n}=L_{n}$, which can be done since $L_{n}$ is finitely generated and $F$ is not). This means that $e_{i}$ is not a noetherian element.

(a) $\Leftrightarrow$ (c). This follows from Corollary 3.5 (or Theorem 3.6) along with [7, Theorem 5], which states that for a rng of the form $\operatorname{fEnd}\left({ }_{R} F\right)$ and $F$ not finitely generated the property of being left $s$-unital is equivalent to $R$ being left noetherian. 


\section{The characterization problem: Main results}

We now consider the general Characterization Problem stated in the introduction. First, we extend slightly our Definition 1.1.

Definition 4.1. Let $A$ be a ring. A family $\left\{e_{i j}\right\}$ of elements of $A$ will be called a generalized family of matric units of $A$ when

(i) $e_{i j} e_{k t}=\delta_{j k} e_{i t}$,

(ii) if $a \in A$ satisfies that $a e_{i i}=0$ for almost all $i \in I$, then we have $a=\sum_{I} a e_{i i}$.

We shall write in this case $e_{i}$ instead of $e_{i i}$. Consider now the family of all the right ideals of $A$ which are right annihilators of idempotents $e_{i}$ (for $i \in I$ ). This family is a subbase of neighborhoods of 0 for a certain right linear topology over $A$. We shall denote this topology by $\Gamma$ and we call it the topology of the matric units of $A$ (assuming that the family $\left\{e_{i j}\right\}$ is known). Also, we denote by $A_{0}$ the left ideal of $A$ generated by the $e_{i}$, that is, the left ideal of $A$ which consists of all the elements $a \in A$ satisfying the hypothesis in condition (ii) above. The following result characterizes the rings which are endomorphism rings of free modules over a given ring $R$.

Theorem 4.2. Let $A$ be a ring, and $\mathscr{R}$ a class of rings. The following conditions are equivalent.

(a) $A \cong \operatorname{End}\left({ }_{R} F\right)$ for some ring $R$ in the class $\mathscr{R}$ and some free left $R$-module $F$.

(b)

(i) $A$ has a generalized family $\left\{e_{i j}\right\} \quad(i, j \in I)$ of matric units, with associate topology $\Gamma$;

(ii) if $A_{0}$ is the left ideal of $A$ generated by the $e_{i}$, then $A_{0}$ is a right ideal of $A$;

(iii) $e_{i} A e_{i}$ is isomorphic to some ring in the class $\mathscr{R}$, for any $i \in I$; and

(iv) $A$ is Hausdorff and complete in the topology $\Gamma$.

Moreover, the family $\left\{e_{i j}\right\}$ may be chosen so that $R \cong \operatorname{End}\left(A e_{i}\right)$ for any $i \in I$.

Proof. (a) $\Rightarrow$ (b). Conditions (i), (ii) and (iii) in (b) follow by taking each $e_{i j}$ to be the projection of the $i$ th component of $F=R^{(I)}$ onto its $j$ th component. This choice made, $A_{0}$ coincides with $\operatorname{fEnd}\left({ }_{R} F\right) \subseteq A$. Now, the condition that $A$ is Hausdorff means that $\bigcap\left\{r_{A}\left(e_{i}\right) \mid i \in I\right\}=0$. But if $a \in A$ satisfies $e_{i} a=0$ for all $i \in I$, then $A_{0} a=0$ from which it follows that $a=0$, for $A$ is plainly $A_{0}$-torsion-free. On the other hand, let $J$ be the family of all the finite subsets of $I$, and for each $j \in J$, with 
$j=\left\{i_{1}, \ldots, i_{r}\right\}$, let us put $f_{j}=e_{i_{1}}+\cdots+e_{i_{r}}, U_{j}=\mu\left(f_{j}\right)=\mu\left(e_{i_{1}}, \ldots, e_{i_{r}}\right)$. Order $J$ by stating $j_{1} \leq j_{2}$ if and only if $j_{1} \subseteq j_{2}$, that is, if and only if $U_{j_{2}} \subseteq U_{j_{1}}$, and let $\left(x_{j}\right)_{j \in J}$ be a Cauchy net in $A$, that is, for each $j \in J$ there is $i \in J$ such that if $k \geq i$, then $x_{k}-x_{i} \in U_{j}$. We are to see that this net has a limit in order to prove that $A$ is complete. Let $\left(u_{t}\right)_{t \in I}$ be the canonical basis of $F=R^{(I)}$, and let $t$ be any element of $I$. Take $t_{0}:=\{t\}$ in $J$, so that $f_{t_{0}}=f_{0}=e_{t}$ and $U_{t_{0}}=U_{0}=r\left(e_{t}\right)$. By hypothesis, there is some $i=i(t)$ such that $x_{k}-x_{i} \in U_{0}=r\left(e_{t}\right)$ for any $k \geq i$; that is, $e_{t} x_{k}=e_{t} x_{i}$. Therefore $u_{t} e_{t} x_{k}=u_{t} x_{k}=u_{t} x_{i}$. Define then $\alpha \in A$ as the endomorphism of $F$ given by $u_{t} \alpha=u_{t} x_{i(t)}$. This is clearly unambiguous, so it only remains to show that $\alpha=\lim \left(x_{j}\right)$. Given any $U_{j}$, for $j \in J$, take $f_{j}$ as above, that is, $f_{j}=e_{j_{1}}+\cdots+e_{j_{s}}$, if $j=\left\{j_{1}, \ldots, j_{s}\right\}$. Then there exists some $i \in J$ such that for each $k \geq i$ we have $x_{k}-x_{i} \in U_{j}$. It follows that $f_{j} x_{k}=f_{j} x_{i}$, and hence $e_{j_{t}} x_{k}=e_{j_{t}} x_{i}$ for any $t, 1 \leq t \leq s$; then $u_{j_{i}} x_{k}=u_{j_{t}} x_{i}$ for these values of $t$. If we now take an index $i_{0}$ in $J$ which is greater than $i$, as well as greater than all the $i\left(j_{t}\right)$, then we will have for each $k \geq i_{0}, u_{j_{t}} x_{k}=u_{j_{t}} x_{i_{0}}$, and $u_{j_{t}} \alpha=u_{j_{t}} x_{i_{0}}$, from which it follows that $u_{j_{t}}\left(x_{k}-\alpha\right)=0$ (for all $t$ in $\{1, \ldots, s\}$ ). Thus $e_{j_{t}}\left(x_{k}-\alpha\right)=0$ and $f_{j}\left(x_{k}-\alpha\right)=0$. So we get that $x_{k}-\alpha \in U_{j}$, for $k \geq i_{0}$. This completes the proof of (iv).

(b) $\Rightarrow$ (a). $\quad A_{0}=\sum_{I} A e_{i}=\bigoplus_{I} A e_{i}$ is a non-degenerate rng in view of (iv). Also, the family $\left\{e_{i j}\right\}$ is a family of matric units of $A_{0}$, so we can apply Proposition 3.4 to obtain that, if $R=e_{i} A e_{i}$ (for any $i \in I$ ) and $F=$ $R^{(I)}$, then $A_{0}$ is isomorphic to a $q$-dense right ideal $B$ of $E_{0}=f \operatorname{End}\left({ }_{R} F\right)$. Moreover, $R$ belongs (up to isomorphism) to the class $\mathscr{R}$, by condition (iii). By Corollary 2.5 we know that there is an isomorphism $\phi: S \rightarrow E=$ $\operatorname{End}\left({ }_{R} F\right)$, where $S$ is the endomorphism ring of $A_{0}$, which coincides with End $\left({ }_{A} A_{0}\right)$, by condition (ii); and this isomorphism $\phi$ takes $A_{0}$ to $B$. By employing again condition (ii) we see that each element $\phi$ takes $A_{0}$ to $B$. By employing again condition (ii) we see that each element of $A$ can be interpreted as an endomorphism of $A_{0}$, so that we have inclusions $A_{0} \subseteq$ $A \subseteq S$. Thus, all that is left to show is that any endomorphism of $A_{0}$ can be given by right multiplication by an element of $A$, so that $A=S \cong \operatorname{End}\left({ }_{R} F\right)$. To this end, let $\alpha \in S$ and put $x_{j}=f_{j} \alpha$ for each $j \in J$, where we keep the notations of the first part of the proof. Then we claim that $\left(x_{j}\right)_{j \in J}$ is a Cauchy net in $A$ : let $j \in J$ and $i \geq j$; then clearly $f_{j} f_{i}=f_{j}$ and hence $x_{i}-x_{j}=f_{i} \alpha-f_{j} \alpha$ satisfies $f_{j}\left(x_{i}-x_{j}\right)=f_{j} \alpha-f_{j} \alpha=0$, from which it follows that $x_{i}-x_{j} \in U_{j}$.

Now, condition (iv) in (b) implies that $\left(x_{j}\right)_{j \in J}$ has a limit, say $a \in A$. 
This entails that for any $j \in J$ there is $i \in J$ such that we have for any $k \geq i, x_{k}-a \in U_{j}$, that is, $f_{j}\left(x_{k}-a\right)=0$, from which we get $f_{j} f_{k} \alpha=f_{j} a$. But if we choose $k$ greater than both $i$ and $j$, we have $f_{j} f_{k} \alpha=f_{j} \alpha=f_{j} a$. Therefore $e_{j} \alpha=e_{j} a$, for all $j \in I$, and thus the endomorphism $\alpha$ of $A_{0}$ is right multiplication by $a$. This shows that $A=S$ and so we are done. The final assertion is clear from the above proof.

As a first application of the theorem we again consider the case which was mentioned in Theorem 3.6, namely that of $R$ being a direct sum, as a left $R$-module, of directly indecomposable left ideals.

Corollary 4.3. Let $A$ be a ring. The following conditions are equivalent.

(a) $A$ is isomorphic to $\operatorname{End}\left({ }_{R} F\right)$ for a ring $R$ such that ${ }_{R} R$ is a direct sum of indecomposable left ideals, and a free $R$-module $F$.

(b)

(i) $A$ has a generalized family of matric units $\left\{e_{i j}\right\}$ such that each $e_{i}$ is a finite idempotent;

(ii) If $A_{0}$ is the left ideal of $A$ generated by the $e_{i}$, then $A_{0}$ is a right ideal of $A$; and

(iii) $A$ is Hausdorff and complete in the topology $\Gamma$ of the matric units of $A,\left\{e_{i j}\right\}$.

Moreover, the family $\left\{e_{i j}\right\}$ can be taken so that $R \cong \operatorname{End}\left(A e_{i}\right)$ for any $i \in I$.

Proof. (a) $\Rightarrow$ (b). This follows from Theorem 4.2 and from the fact that $R$ is then isomorphic to $\operatorname{End}\left(A e_{i}\right)$ for any $i \in I$, so that it is a direct sum of finitely many indecomposable direct summands and hence each $e_{i}$ is a finite idempotent.

(b) $\Rightarrow$ (a). Again by Theorem 4.2, (b) implies the existence of an isomorphism $A \cong \operatorname{End}\left({ }_{R} F\right)$, with $R \cong \operatorname{End}\left(A e_{i}\right)$ through the equivalence $R$-mod $\cong A_{0}$-mod. Then the fact that $A e_{i}$ is a finite direct sum of indecomposable modules, by the finiteness condition on $e_{i}$, implies that the same happens to $R$.

REMARK 4.4. In [3], a ring $R$ is said to be an $I F$-ring when every nonzero summand of a free $R$-module is indecomposable if and only if it is isomorphic to ${ }_{R} R$. Corollary 4.3 can be obviously applied to $I F$-rings: if $R$ is such, then one can choose the family $\left\{e_{i j}\right\}$ in the natural manner of the proof of Theorem 4.2, and then $\Gamma$ is the finite topology on $A \cong \operatorname{End}\left({ }_{R} F\right)$, $A_{0}$ contains all the finite idempotents and each $e_{i}$ is primitive. Thus it is easy to obtain from this [13, Theorem 3.1], for if the conditions therein hold, then the direct sum $\bigoplus_{I} E e_{i}$ (in the notation of [13]) may be written as $\bigoplus_{j} E u_{j}$ for primitive idempotents $u_{j}$ and hence one can see that (b) of Corollary 4.3 also holds. 
As in Section 3, we also consider the particular case in which $R$ is left noetherian. We then have the following result.

COROLlary 4.5. Let $A$ be a ring. The following conditions are equivalent.

(a) There is a left noetherian ring $R$ and a non-finitely generated free left $R$-module $F$ such that $A \cong \operatorname{End}\left({ }_{R} F\right)$.

(b)

(i) $A$ has an infinite generalized family of matric units $\left\{e_{i j}\right\}$ such that each $e_{i}$ is a noetherian idempotent;

(ii) if $A_{0}$ is the left ideal of $A$ generated by the $\left\{e_{i}\right\}$, then $A_{0}$ is a right ideal of $A$; and

(iii) $A$ is Hausdorff and complete in the topology $\Gamma$ of the matric units $\left\{e_{i j}\right\}$.

(c)

(i) $A$ has an infinite generalized family of matric units $\left\{e_{i j}\right\}$;

(ii) if $A_{0}$ is the left ideal of $A$ generated by the $\left\{e_{i}\right\}$, then $A_{0}$ is a right ideal of $A$ which is left s-unital as a rng; and

(iii) $A$ is Hausdorff and complete in the topology $\Gamma$ of the matric units $\left\{e_{i j}\right\}$.

PROof. This is again a consequence of Theorem 4.2 along with Corollary 3.7.

It should be noted (as Liebert does in [13]) that the older result of Wolfson [20, Theorem 7.5] about the characterization of the endomorphism rings of (free) modules over division rings is not a corollary of Liebert's. However, a modification (for a special case) of the categorical arguments given in the proof of Theorem 4.2 allows us to include also Wolfson's result under a more general frame. Recall that a ring $R$ is said to be left Kasch when ${ }_{R} R$ cogenerates all the simple left $R$-modules. Also, a left ideal $I$ of a ring $R$ is said to be dense when the left ideal $(I: a)$ (where $(I: a)=\{r \in R \mid r a \in I\}$ ) has no nonzero right annihilators for any $a \in R$. To obtain our next theorem we need the following lemma, which is an easy consequence of Lemma 2.3 and Proposition 3.4.

LEMMA 4.6. Let $A$ be a ring with a generalized family of matric units, $\left\{e_{i j}\right\} \quad(i, j \in I)$ and assume that $A_{0}=\sum_{I} A e_{i}$ is a two-sided ideal of $A$ and $A$ is $A_{0}$-torsion-free. Put $R=\operatorname{End}\left({ }_{A} A e_{i}\right)$ for any $i \in I,{ }_{R} F=R^{(I)}$ and let $\mathscr{F}$ be the left Gabriel topology of $A$ given by $\mathscr{F}=\left\{I \subseteq{ }_{A} A \mid A_{0} \subseteq I\right\}$. Then there is an equivalence of categories between $(A, \mathscr{F})-\bmod$ and $R-\bmod$ and, if $A_{\mathscr{G}}$ is the localization of $A$ in $(A, \mathscr{F})$-mod, then $A_{\mathscr{G}} \cong \operatorname{End}\left({ }_{R} F\right)$. 
Proof. Since $A$ is $A_{0}$-torsion-free (that is, $A_{0}$ is a faithful right ideal of $A$ ), Lemma 2.3 tells us that there is an equivalence $L$ from $(A, \mathscr{F})$-mod to $A_{0}$-mod such that $L\left(A_{\mathscr{g}}\right)=A_{0} A_{\mathscr{F}}=A_{0} A=A_{0}$. Now $A_{0}$ satisfies the hypothesis of Proposition 3.4 and hence $A_{0}$ is isomorphic to a $q$-dense right ideal of $E_{0}=\operatorname{fEnd}\left({ }_{R} F\right)$, with $R$ and ${ }_{R} F$ as above. By Theorem 2.4, $A_{0}$ mod is equivalent to $R$-mod, with $A_{0}$ corresponding to ${ }_{R} F$, by Corollary 2.5. Thus $(A, \mathscr{F})-\bmod$ is equivalent to $R-\bmod$ and $A_{\mathscr{F}}=\operatorname{End}\left(A_{\mathscr{F}}\right) \cong$ $\operatorname{End}\left({ }_{R} F\right)$.

THEOREM 4.7. Let $A$ be a ring. The following conditions are equivalent.

(a) There is a left Kasch ring $R$ and a free left $R$-module $F$ such that $A \cong \operatorname{End}\left({ }_{R} F\right)$.

(b)

(i) $A$ has a generalized family of matric units $\left\{e_{i j}\right\}$;

(ii) if $A_{0}$ is the left ideal generated by the $\left\{e_{i}\right\}$, then $A_{0}$ is the smallest dense left ideal of $A$;

(iii) if $J$ is a left ideal of $A$ which is maximal with respect to the property of not being dense, then ${ }_{A}(J) \neq 0$; and

(iv) $A$ is its own maximal left ring of quotients.

Proof. (a) $\Rightarrow$ (b). Take each $e_{i}$ as the canonical projection of ${ }_{R} F=R^{(I)}$ onto its $i$ th component. Then, if we put $A=\operatorname{End}\left({ }_{R} F\right), A_{0}=\sum_{I} A e_{i}=$ fEnd $\left({ }_{R} F\right)$, we see that (i) holds. Assume that $J$ is a left-ideal of $A=$ End $\left({ }_{R} F\right)$ such that $r_{A}(J)=0$ and put $L=\sum_{\sigma \in J} \operatorname{Im} \sigma$. Then the above assumption implies that $\operatorname{Hom}_{R}(F / L, F)=0$ and it follows from the fact that $R$ is left Kasch that $e_{i}(L)=\operatorname{Im} e_{i}$ for each $i \in I$. Suppose now that $J$ is a dense left ideal and put $K=K(i)=\left(J: e_{i}\right)$ for each $i \in I$, so that $r_{A}(K)=0$. We have therefore that $e_{j}\left(L_{i}\right)=\operatorname{Im} e_{j}$, if $L_{i}=\sum_{\sigma \in K} \operatorname{Im} \sigma$ and hence $e_{i}\left(L_{i}\right)=\operatorname{Im} e_{i}$ for any $i \in I$. But if $s \in K$, then $s e_{i} \in J$, so that $e_{i}(\operatorname{Im} s) \subseteq L$ and $e_{i}\left(L_{i}\right)=\operatorname{Im} e_{i} \subseteq L$. Since $\sum_{I} \operatorname{Im} e_{i}=F$, we get $L=F$ and $A_{0} \subseteq J$, by $[8, \mathrm{p} .93]$. This proves that $A_{0}$ is the smallest dense left ideal.

To verify (iii), let $J$ be maximal with respect to the property that $J$ is not dense, and put $M=\sum_{\sigma \in J} \operatorname{Im} \sigma$. Then $M \neq F$, because $J$ does not contain $A_{0}$; and $J=\operatorname{Hom}_{R}(F, M)$ with $M$ a maximal submodule of $F$, because of the maximality of $J$. Since $R$ is left Kasch, $\operatorname{Hom}_{R}(F / M, F) \neq 0$, from which we deduce that ${ }^{\prime}{ }_{A}(J) \neq 0$, proving (iii). Finally, the maximal left ring of quotients of $A$ is in this case, $A_{\mathscr{F}}=\operatorname{End}_{A}\left(A_{0}\right) \cong A$ by $[18$, Corollary IX.2.9].

(b) $\Rightarrow$ (a). Since $A_{0}$ is the smallest dense left ideal of $A, A_{0}$ must be a two-sided ideal in $A$; also, the condition that $A_{0}$ is dense implies that $A$ is 
$A_{0}$-torsion-free. Thus we obtain from Lemma 4.6 that $A_{\mathscr{F}} \cong \operatorname{End}\left({ }_{R} F\right)=E$. Since $F$ is precisely the left Gabriel topology of $A$ consisting of all the dense left ideals [18, Proposition VI.6.4], condition (iv) implies that $A \cong E$. To complete the proof we have to show that $R$ is left Kasch: assume not, and let $F / L$ be a simple module such that $\operatorname{Hom}_{R}(F / L, F)=0$; then $J=$ $\operatorname{Hom}_{R}(F, L)$, viewed as a left ideal of $A$, satisfies $r_{A}(J)=0$, while, obviously, every left ideal $K$ of $A$ properly containing $J$ verifies $\sum_{s \in K} \operatorname{Im} s=F$ and hence $A_{0} \subseteq K$ by [8, p. 93]. But this contradicts condition (iii) above.

The following special cases are particularly simple to state.

COROLlaRy 4.8. Let $A$ be a ring. The following assertions are equivalent.

(a) $A$ is isomorphic to $\operatorname{End}\left({ }_{R} F\right)$ for some quasi-Frobenius ring $R$ and non-finitely generated free module $F$.

(b)

(i) $A$ has an infinite generalized family of matric units, $\left\{e_{i j}\right\}$;

(ii) if $A_{0}$ is the left ideal of $A$ generated by the $\left\{e_{i}\right\}$, then $A_{0}$ is a right ideal of $A$ and $A$ is $A_{0}$-torsion-free; and

(iii) $A$ is left self-injective.

Proof. (a) $\Rightarrow$ (b). Conditions (i) and (ii) are clear from Theorem 4.7, while (iii) is well-known (see, for instance, [16, Proposition 4]).

(b) $\Rightarrow$ (a). By using Lemma 4.6 we obtain from (i) and (ii) that $A_{\mathscr{F}} \cong$ End $\left({ }_{R} F\right)$ for ${ }_{R} F$ free and non-finitely generated. By (iii), $A \cong A_{\mathscr{F}}[18$, page 198]. Again [16, Proposition 4] shows that $R$ is quasi-Frobenius.

COROLLARY 4.9. Let $A$ be a ring. The following conditions are equivalent.

(a) $A$ is isomorphic to $\operatorname{End}\left({ }_{D} V\right)$ for some division ring $D$ and $D$-vector space $V$.

(b)

(i) $A$ has a smallest dense left ideal $A_{0}$;

(ii) $A_{0}$ is a quasi-simple rng; and

(iii) $A$ is its own maximal left ring of quotients.

Proof. (a) $\Rightarrow$ (b) is clear.

(b) $\Rightarrow$ (a). By (i), $A$ is $A_{0}$-torsion-free and by (iii), $A \cong \operatorname{End}\left({ }_{A} A_{0}\right) \cong$ End $\left(A_{0}\right)$ (as an $A_{0}$-module), in view of [18, Corollary IX.2.9]. By Proposition 2.8, condition (ii) implies that $A_{0}$ is $q$-dense in some $\operatorname{End}\left({ }_{D} V\right)$. Since $A_{0}$ is idempotent by (i), Theorem 2.4 and Corollary 2.5 imply that $A \cong \operatorname{End}\left(A_{0}\right) \cong \operatorname{End}\left({ }_{D} V\right)$.

REMARK 4.10. We point out that Corollary 4.9 can also be obtained as a consequence of [18, Corollary XII.1.5]. 
We return now to the question of how [20, Theorem 7.5] is related to our theory, namely to Corollary 4.9. Essentially, [20, Theorem 7.5] states that if $A_{0}$ is $\operatorname{fEnd}\left({ }_{D} V\right), E=\operatorname{End}\left({ }_{D} V\right)$ and $A$ is an intermediate subring, that is, $A_{0} \subseteq A \subseteq E$, then $A=E$ if and only if the sum of two left annihilators in $A$ is again a left annihilator. These hypotheses imply that $A$ is left non-singular (so that the dense topology $\mathscr{D}$ of $A$ coincides with the Goldie topology) and that $E$ is the maximal left ring of quotients of $A$. By [18, Proposition XII.4.7] and [20, Theorem 6.2] the essentially closed left ideals of $A$ (which are the $\mathscr{D}$-saturated left ideals) are exactly the left annihilators. So we want to see that, under the above hypotheses, $A$ coincides with $E$ if and only if the sum of $\mathscr{D}$-saturated left ideals of $A$ is $\mathscr{D}$-saturated. If $A=E$, this condition holds because $E$ is left self-injective. For the converse, let $e$ be an idempotent of $E, X_{1}=E e, X_{2}=E(1-e), Y_{i}=A \cap X_{i}$. Then the $Y_{i}$ are $\mathscr{D}$-saturated in $A$, whence $Y_{1}+Y_{2}$ is $\mathscr{D}$-saturated. But $X_{i} / Y_{i}$ is $\mathscr{D}$ torsion, so $\left(X_{1}+X_{2}\right) /\left(Y_{1}+Y_{2}\right)$ is $\mathscr{D}$-torsion and so is $A /\left(Y_{1}+Y_{2}\right)$. Therefore $Y_{1}+Y_{2}=A$ and hence each idempotent of $E$ belongs to $A$. Since $E$ is a regular ring, we have that $A=E$.

\section{Acknowledgement}

The author is grateful to the referee for several suggestions which contributed to improve this paper.

\section{References}

[1] F. W. Anderson and K. R. Fuller, Rings and categories of modules, (Springer-Verlag, New York, 1973).

[2] G. M. Brodskii, 'Annihilator conditions in endomorphism rings of modules', Math. Notes 16 (1974), 1153-1158.

[3] W. N. Franzsen and P. Schultz, 'The endomorphism ring of a locally free module', $J$. Austral. Math. Soc. Ser. A 35 (1983), 308-326.

[4] K. R. Fuller, 'Density and equivalence', J. Algebra 29 (1974), 528-550.

[5] L. Fuchs, Infinite abelian groups, Vol. 1 (Academic Press, New York, 1970).

[6] J. L. Garcia, 'Morita-like equivalences of categories of modules', preprint.

[7] J. L. Garcia, 'The finite column matrix ring of a ring', Proceedings of the First SpanishBelgian Week on Algebra and Geometry, eds. J. L. Bueso, M. I. Segura, A. Verschoren, R. U. C. A., Antwerpen, 1988, pp. 64-74.

[8] J. L. García and J. L. Gómez Pardo, 'On endomorphism rings of quasi-projective modules', Math. Z. 196 (1987), 87-108.

[9] J. L. Garcia and M. Saorín, 'Endomorphism rings and category equivalences', J. Algebra, 127 (1989), 324-350. 
[10] N. Jacobson, Structure of rings, (AMS Colloq. Publ., Vol. 37, Amer. Math. Soc., Providence, R.I., 1968).

[11] W. Liebert, 'Characterization of the endomorphism rings of divisible torsion modules and reduced complete torsion-free modules over complete discrete valuation rings', $\mathrm{Pa}$ cific J. Math. 37 (1971), 141-170.

[12] W. Liebert, 'Endomorphism rings of reduced torsion-free modules over complete discrete valuation rings', Trans. Amer. Math. Soc. 169 (1972), 347-363.

[13] W. Liebert, 'Endomorphism rings of free modules over principal ideal domains', Duke Math. J. 41 (1974), 323-328.

[14] C. Metelli and L. Salce, 'The endomorphism ring of an abelian torsion-free homogeneous separable group', Arch. Math. (Basel) 26 (1975), 480-485.

[15] B. J. Müller, 'The quotient category of a Morita context', J. Algebra 28 (1974), 389-407.

[16] B. L. Osofsky, 'Some properties of rings reflected in endomorphism rings of free modules', in Contemporary Math., Vol. 13, pp. 179-181, (Amer. Math. Soc., Providence, R.I., 1982).

[17] L. H. Rowen, Ring theory, Vol. I, (Academic Press, Boston, Mass., 1988).

[18] B. Stenström, Rings of quotients, (Springer-Verlag, Berlin, 1975).

[19] H. Tominaga, 'On s-unital rings', Math. J. Okayama Univ. 18 (1976), 117-134.

[20] K. G. Wolfson, 'An ideal-theoretic characterization of the ring of all linear transformations', Amer. J. Math. 75 (1953), 358-386.

[21] B. Zimmermann, 'Endomorphism rings of self-generators', Algebra Berichte 27, Math. Inst. Univ. München (1975).

\section{Universidad de Murcia \\ 30001 Murcia \\ Spain}

